\title{
Challenging nostalgia and performance metrics in baseball
}

\author{
Daniel J. Eck \\ Department of Biostatistics, Yale University, New Haven, CT, 06510. \\ daniel.eckeyale.edu
}

\section{Introduction}

It is easy to be blown away by the accomplishments of great old time baseball players when you look at their raw or advanced baseball statistics. These players produced mind-boggling numbers. For example, see Babe Ruth's batting average and pitching numbers, Ty Cobb's 1911 season, Walter Johnson's 1913 season, Tris Speaker's 1916 season, Rogers Hornsby's 1925 season, and Lou Gehrig's 1931 season. The statistical feats achieved by these players (and others) far surpass the statistics that recent and current players produce. At first glance it seems that players from the old eras were vastly superior to the players in more modern eras, but is this true? In this paper, we investigate whether baseball players from earlier eras of professional baseball are overrepresented among the game's all-time greatest players according to popular opinion, performance metrics, and expert opinion. We define baseball players from earlier eras to be those that started their MLB careers in the 1950 season or before. This year is chosen because it coincides with the decennial US Census and is close to 1947, the year in which baseball became integrated.

In this paper we do not compare baseball players via their statistical accomplishments. Such measures exhibit era biases that are confounded with actual performance. Consider the single season homerun record as an example. Before Babe Ruth, the single season homerun record was 27 by Ned Williams in 1884. Babe Ruth broke this record in 1919 when he hit 29 homeruns. He subsequently destroyed his own record in the following 1920 season when he hit 54 homeruns. The runner up in 1920 finished the season with a grand total of 15 homeruns. At this point in time homerun hitting was not an integral part of a batter's approach. This has changed. Now, we often see multiple batters reach at least 30-40 homeruns within one season and a 50 homerun season is not a rare occurrence. In the 1920s, Babe Ruth stood head and shoulders above his peers due to a combination of his innate talent and circumstance. His approach was quickly emulated and became widely adopted. However, Ruth's accomplishments as a homerun hitter would not stand out nearly as much if he played today and put up similar homerun totals. The example of homeruns hit by Babe Ruth and the impact they had relative to his peers represents a case where adjustment towards a peer-derived 
baseline fails across eras. No one reasonably expects 1920 Babe Ruth to hit more than three times the amount of homeruns hit by the second best homerun hitter if the 1920 version of Babe Ruth played today. This is far from an isolated case.

There are several statistical approaches currently used to compare baseball players across eras. These include wins above replacement as calculated by baseball reference (bWAR), wins above replacement as calculated by fangraphs (fWAR), adjusted OPS+, adjusted ERA+, era-adjusted detrending (Petersen et al., 2011), computing normal scores as in Jim Albert's work on a Baseball Statistics Course in the Journal of Statistics Education, and era bridging (Berry et al., 1999). A number of these are touted to be season adjusted and the remainder are widely understood to have the same effect. In one way or another all of these statistical approaches compare the accomplishments of players within one season to a baseline that is computed from statistical data within that same season. This method of player comparison ignores talent discrepancies that exist across seasons as noted by Stephen J. Gould in numerous lectures and papers. Currently, there is no definitive quantitative or qualitative basis for comparing these baselines, which are used to form intra-season player comparisons, across seasons. These methods therefore fail to properly compare players across eras of baseball despite the claim that they are season adjusted.

Worse still is that these approaches exhibit a favorable bias towards baseball players who played in earlier seasons (Schmidt and Berri, 2005). We explore this bias from two separate theoretical perspectives underlying how baseball players from different eras would actually compete against each other. The first perspective is that players would teleport across eras to compete against each other. From this perspective, the players from earlier eras are at a competitive disadvantage because, on average, baseball players have gotten better as time has progressed. Specifically, it is widely acknowledged that fastball velocity, pitch repertoire, training methods, and management strategies have all improved over time. We do not find the teleportation perspective to be of much interest for these reasons. The second perspective is that a player from one era could adapt naturally to the game conditions of another era if they grew up in that time. This line of thinking is challenging to current statistical methodology because adjustment to a peer-derived baseline no longer makes sense. Even in light of these challenges with the second perspective, we find that the players from earlier eras are overrepresented among baseball's all time greats. We justify our findings through the consideration of population dynamics which have changed drastically over time. 


\section{Data}

The MLB eligible population is not well-defined. As a proxy, we can say that the MLB eligible population is the decennial count of males aged 20-29 that are living in the United States (US) and Canada. Baseball was segregated on racial grounds until 1947. As a result, African American and Hispanic American population counts in the US and Canada are added to our dataset starting in 1960. The year 1960 is chosen because the integration of the MLB was slow as noted in Armour's work on the integration of baseball in the Society for American Baseball Research.

Players from Central and South American countries and the Caribbean islands were also targets of discrimination. We have added data from these countries to the MLB eligible population starting in 1960: Mexico, the Dominican Republic, Venezuela, Cuba, Panama, Puerto Rico, Netherlands Antilles, Aruba, Honduras, Jamaica, the Bahamas, Peru, Columbia, Nicaragua, and the United States Virgin Islands. In the mid to late 1990s, the MLB and minors saw an influx of Asian baseball players from Japan, South Korea, Taiwan, and the Philippines. We have added the populations of these countries to the MLB eligible population starting in 2000. In 2010, the MLB established a national training center in Brazil as noted in Loré's work on the popularity of baseball in Brazil in the Culture Trip. Therefore, we have included the Brazilian population of 20-24 year old men into our MLB eligible population starting in 2010. We estimate that the 2011-2015 MLB eligible population is half of the MLB eligible population counted in the 2010 decennial Censuses. We expect that this underestimates the actual 2011-2015 MLB eligible population since we have observed a constant increase in the overall MLB eligible population as time increases.

The MLB eligible population is displayed in Table 1. The cumulative proportion means that at each era, the population of the previous eras is also included. As an example of how to interpret this dataset, consider the year 1950. There were 11.59 million males aged 20-29. The proportion of the historical MLB eligible population that existed at or before 1950 is 0.187 .

\section{The greats}

To determine which players are the all-time greatest players, we consult four lists which reflect popular opinion, performance metrics, and expert opinion that purport to determine the greatest players. The first list is compiled by Ranker, which is constructed entirely from popular opinion as determined by up and down 


\begin{tabular}{lccc}
\hline & year & population & cumulative population proportion \\
\hline 1 & 1880 & 4.440 & 0.013 \\
2 & 1890 & 5.010 & 0.027 \\
3 & 1900 & 5.580 & 0.043 \\
4 & 1910 & 8.560 & 0.068 \\
5 & 1920 & 8.930 & 0.093 \\
6 & 1930 & 9.920 & 0.122 \\
7 & 1940 & 11.130 & 0.154 \\
8 & 1950 & 11.590 & 0.187 \\
9 & 1960 & 18.420 & 0.240 \\
10 & 1970 & 24.490 & 0.310 \\
11 & 1980 & 33.930 & 0.407 \\
12 & 1990 & 37.460 & 0.515 \\
13 & 2000 & 60.660 & 0.689 \\
14 & 2010 & 72.270 & 0.896 \\
15 & 2015 & 36.140 & 1.000 \\
\hline
\end{tabular}

Table 1: Eligible MLB population throughout the years. The first column indicates the year, the second column indicates the estimated MLB eligible population size (in millions), and the third column indicates the proportion of the MLB eligible population in row $\mathrm{x}$ that was eligbile at or before row $\mathrm{x}$.

votes. The second and third lists rank players by highest career WAR as calculated by baseball reference and fangraphs, respectively. The fourth list is a ranking from ESPN and is based on expert opinion and statistics.

The rankings for all four lists are given in Table 2. As an example of the information contained in Table 2 consider the greatest players of all time according to ESPN displayed in the fourth column. We see that 5 players that started their careers before 1950 are in the top 10 all time and 11 players that started their careers before 1950 are in the top 25 all time. When the MLB eligible population is considered, it appears that the players from the earlier eras are overrepresented in this particular list.

\section{Statistical evidence}

We now provide evidence that the top 10 and top 25 lists displayed in Table 2 overrepresent players who started their careers before 1950 . We require two assumptions for the validity of our calculations which we will explore in detail in the next Section. These assumptions are:

- First, we assume that innate talent is uniformly distributed over the MLB eligible population over the different eras.

- Second, we assume that the outside competition to the MLB available by other sports leagues after 


\begin{tabular}{|c|c|c|c|c|}
\hline rank & Ranker & bWAR & fWAR & ESPN \\
\hline 1 & Babe Ruth & Babe Ruth & Babe Ruth & Babe Ruth \\
\hline 2 & Ty Cobb & Cy Young & Barry Bonds & Willie Mays \\
\hline 3 & Lou Gehrig & Walter Johnson & Willie Mays & Barry Bonds \\
\hline 4 & Ted Williams & Barry Bonds & Ty Cobb & Ted Williams \\
\hline 5 & Stan Musial & Willie Mays & Honus Wagner & Hank Aaron \\
\hline 6 & Willie Mays & Ty Cobb & Hank Aaron & Ty Cobb \\
\hline 7 & Hank Aaron & Hank Aaron & Roger Clemens & Roger Clemens \\
\hline 8 & Mickey Mantle & Roger Clemens & Cy Young & Stan Musial \\
\hline 9 & Rogers Hornsby & Tris Speaker & Tris Speaker & Mickey Mantle \\
\hline 10 & Honus Wagner & Honus Wagner & Ted Williams & Honus Wagner \\
\hline 11 & Cy Young & Stan Musial & Rogers Hornsby & Lou Gehrig \\
\hline 12 & Walter Johnson & Rogers Hornsby & Stan Musial & Walter Johnson \\
\hline 13 & Joe Dimaggio & Eddie Collins & Eddie Collins & Greg Maddux \\
\hline 14 & Sandy Koufax & Ted Williams & Walter Johsnon & Rickey Henderson \\
\hline 15 & Ken Griffey Jr. & Pete Alexander & Greg Maddux & Rogers Hornsby \\
\hline 16 & Jimmie Foxx & Alex Rodriguez & Lou Gehrig & Mike Schmidt \\
\hline 17 & Tris Speaker & Kid Nichols & Alex Rodriguez & Cy Young \\
\hline 18 & Joe Jackson & Lou Gehrig & Mickey Mantle & Joe Morgan \\
\hline 19 & Mike Schmidt & Rickey Henderson & Randy Johnson & Joe Dimaggio \\
\hline 20 & Nolan Ryan & Mickey Mantle & Mel Ott & Frank Robinson \\
\hline 21 & Christy Mathewson & Tom Seaver & Nolan Ryan & Randy Johnson \\
\hline 22 & Roberto Clemente & Mel Ott & Mike Schmidt & Tom Seaver \\
\hline 23 & Albert Pujols & Nap Lajoie & Rickey Henderson & Alex Rodriguez \\
\hline 24 & Cap Anson & Frank Robinson & Frank Robinson & Tris Speaker \\
\hline 25 & Greg Maddux & Mike Schmidt & Burt Blyleven & Steve Carlton \\
\hline pre-1950 in top 10 & $7 / 10$ & $6 / 10$ & $6 / 10$ & $5 / 10$ \\
\hline pre-1950 in top 25 & $15 / 25$ & $15 / 25$ & $12 / 25$ & $11 / 25$ \\
\hline
\end{tabular}

Table 2: Lists of the top 25 greatest baseball players to ever play in the MLB according to Ranker.com (1st column), bWAR (2nd column), fWAR (3rd column), and ESPN (4th column). Players that started their career before 1950 are indicated in bold. The last two rows count the number of players that started their careers before 1950 in each of the top 10 and top 25 lists respectively.

1950 is offset by the increased salary incentives received by MLB players.

With these assumptions in mind we calculate the probability that at least $\mathrm{x}$ people from each top 10 and top 25 list in Table 2 started their career before 1950 using the proportion depicted in Table 1. Consider the bWAR list for example. According to bWAR, we see that 6 of the top 10 players started their careers before 1950. From Table 1 we see that the proportion of the MLB eligible population that played at or before 1950 was approximately 0.187 . We then calculate the probability that one would expect to observe 6 or more individuals in a top 10 list from that time period where the chance of observing each individual is about 
0.187. We calculate this probability using the Binomial distribution. We perform the same type of extreme event calculation for each top 10 and top 25 list depicted in Table 2 . The results are provided in Table 3

\begin{tabular}{lllll}
\hline & Ranker & bWAR & fWAR & ESPN \\
\hline probability of extreme event in top 10 list & 0.000562 & 0.00448 & 0.00448 & 0.0249 \\
probability of extreme event in top 25 list & 0.0000057 & 0.0000057 & 0.000826 & 0.00322 \\
chance of extreme event in top 10 list & 1 in 1780 & 1 in 223 & 1 in 223 & 1 in 40 \\
chance of extreme event in top 25 list & 1 in 174816 & 1 in 174816 & 1 in 1210 & 1 in 310 \\
\hline
\end{tabular}

Table 3: The probability and chance ( 1 in 1/probability) of each extreme event calculation corresponding to the four lists in Table 2 ,

As an example of how to interpret the results of Table 3, continue with bWAR's top 10 list. Table 3 shows that the probability of observing 6 or more players that started their careers at or before 1950 of the top 10 all time players, based on population dynamics, is about 0.00448 (a chance of 1 in 223). The same interpretation applies to remainder of Table 3 . The results provided in Table 3 present overwhelming evidence that players who started their careers before 1950 are overrepresented in top 10 and top 25 lists from the perspectives of fans, analytic assessment of performance, and experts' rankings.

\section{Assumptions and Sensitivity Analysis}

The results in Table 3 are valid under the two assumptions provided in the previous Section. In the first of these assumptions we specify that innate talent is evenly dispersed across eras. We do not fully believe that the first assumption holds because the distribution of innate talent has improved over time as the MLB eligible population has expanded as noted by Stephen J. Gould, Christina Kahrl at ESPN, and in Martin B. Schmidt and David J. Berri's work on concentration of baseball talent in the Journal of Sports Economics. This suggests that the probabilities displayed in Table 3 are conservative.

With respect to the second assumption, we note that the National Basketball Association (NBA) and the National Football League (NFL) started in 1946 and 1920 respectively with both sports greatly rising in popularity since the inception of their respective professional leagues. Soccer and hockey have also risen in popularity in the United States. That being said, it is widely known that MLB salaries have substantially increased. For example, the 1967 census lists the median US household income as $\$ 7,200$. The minimum MLB salary at that time was $\$ 6,000$ as noted by the LA Times sports writer Bill Shaiken in a piece titled "A look at how Major League Baseball salaries have grown by more than 20,000\% the last 50 years." In short, 
baseball players made far less than they do today relative to the general US population and it is unlikely that one could consider playing professional baseball to be a lucrative career in the earlier eras. These figures offer evidence that while other professional leagues may have drawn from the MLB eligible talent pool, salary incentives have led to an increase in the overall quality of MLB players.

Though we cannot confirm this theory with absolute certainty, at worst, our our second assumption suffers some modest violations. To account for this possibility we consider a sensitivity analysis applied to the findings in Table 3. We weight the decennial populations displayed in Table 1 to reflect the overall interest that the US population has had in baseball over time irrespective of salary increases based on Gallup polling data. The four weighting regimes that we consider are given in Table 4 below. These regimes serve as proxies for the proportion of the MLB eligible population thought to strive towards a career in professional baseball. In an effort to be conservative, we have deliberately placed greater weight on the time periods before 1940 for each weighting regime because no polling data is available. We do not expect the MLB eligible population before 1940 to be as high as our weighting regimes suggest because of relatively modest baseball attendance figures in early eras of baseball, non existence of the radio prior to 1920, the dead-ball era, and low compensation.

David W. Moore and Joseph Carroll's Gallup article entitled "Baseball Fan Numbers Steady, But Decline May Be Pending" shows that interest in baseball has remained steady since 1937, at approximately 40\%. Consistent with this benchmark, the first and second weighting regimes (w1 and w2) conservatively place 0.50 and 0.60 weights, repectively, on fan interest prior to 1940. The third weighting regime (w3), constructed from the Gallup polling data (https://news.gallup.com/poll/4735/sports.aspx), reflects the proportion of the US population who listed baseball as their favorite sport. The appropriateness of this regime is intuitively questionable because some people play baseball even if it is not their favorite sport and the weight placed on pre-1940 years is very high. The fourth weighting regime (w4) is the average of w2 and w3.

These weights are obtained from survey data from the US because similar data is unavailable from other countries. We applied these same weights to all of the other countries, even though interest in baseball in these other countries is thought to either be on par with or much greater than the US. Therefore our weighting regimes address, and in fact, overcompensate for any potential shortcomings of no weighting.

Table 5 shows the effect of these weighting regimes as applied to the results in Table 3 . The conclusions from weighting populations with respect to w1, w2, and w4 in Table 5 are largely consistent with those in 


\begin{tabular}{rrrrrrrrrrrrrrrr}
\hline & 1880 & 1890 & 1900 & 1910 & 1920 & 1930 & 1940 & 1950 & 1960 & 1970 & 1980 & 1990 & 2000 & 2010 & 2015 \\
\hline w1 & 0.50 & 0.50 & 0.50 & 0.50 & 0.50 & 0.50 & 0.40 & 0.40 & 0.40 & 0.40 & 0.40 & 0.40 & 0.40 & 0.40 & 0.40 \\
w2 & 0.60 & 0.60 & 0.60 & 0.60 & 0.60 & 0.60 & 0.40 & 0.40 & 0.40 & 0.40 & 0.40 & 0.40 & 0.40 & 0.40 & 0.40 \\
w3 & 0.40 & 0.40 & 0.40 & 0.40 & 0.40 & 0.40 & 0.35 & 0.38 & 0.34 & 0.28 & 0.16 & 0.16 & 0.13 & 0.12 & 0.10 \\
w4 & 0.50 & 0.50 & 0.50 & 0.50 & 0.50 & 0.50 & 0.38 & 0.39 & 0.37 & 0.34 & 0.28 & 0.28 & 0.27 & 0.26 & 0.25 \\
\hline
\end{tabular}

Table 4: Weighting regimes.

\begin{tabular}{|c|c|c|c|c|c|}
\hline weight & & Ranker & bWAR & fWAR & ESPN \\
\hline \multirow[t]{4}{*}{ w1 } & probability of extreme event in top 10 list & 0.00121 & 0.00839 & 0.00839 & 0.0406 \\
\hline & probability of extreme event in top 25 list & 0.0000267 & 0.0000267 & 0.0025 & 0.00845 \\
\hline & chance of extreme event in top 10 list & 1 in 824 & 1 in 119 & 1 in 119 & 1 in 25 \\
\hline & chance of extreme event in top 25 list & 1 in 37519 & 1 in 37519 & 1 in 401 & 1 in 118 \\
\hline \multirow[t]{4}{*}{ w2 } & probability of extreme event in top 10 list & 0.0023 & 0.0141 & 0.0141 & 0.0604 \\
\hline & probability of extreme event in top 25 list & 0.0000944 & 0.0000944 & 0.00608 & 0.0182 \\
\hline & chance of extreme event in top 10 list & 1 in 434 & 1 in 71 & 1 in 71 & 1 in 17 \\
\hline & chance of extreme event in top 25 list & 1 in 10595 & 1 in 10595 & 1 in 164 & 1 in 55 \\
\hline \multirow[t]{4}{*}{ w3 } & probability of extreme event in top 10 list & 0.0311 & 0.109 & 0.109 & 0.273 \\
\hline & probability of extreme event in top 25 list & 0.0128 & 0.0128 & 0.152 & 0.266 \\
\hline & chance of extreme event in top 10 list & 1 in 32 & 1 in 9 & 1 in 9 & 1 in 3.7 \\
\hline & chance of extreme event in top 25 list & 1 in 78 & 1 in 78 & 1 in 6.6 & 1 in 3.8 \\
\hline \multirow[t]{4}{*}{ w4 } & probability of extreme event in top 10 list & 0.00622 & 0.0311 & 0.0311 & 0.11 \\
\hline & probability of extreme event in top 25 list & 0.000649 & 0.000649 & 0.0227 & 0.0561 \\
\hline & chance of extreme event in top 10 list & 1 in 161 & 1 in 32 & 1 in 32 & 1 in 9.1 \\
\hline & chance of extreme event in top 25 list & 1 in 1542 & 1 in 1542 & 1 in 44 & 1 in 18 \\
\hline
\end{tabular}

Table 5: The probability and chance ( 1 in 1/probability, rounded) of each extreme event calculation corresponding to the four lists in Table 2 after the MLB eligible population in Table 1 is weighted according to the four conservative weighting regimes.

Table 3. The third weighting regime presents some conflicting conclusions. When weighting populations with respect to w3 we see that popular opinion and bWAR overrepresent players who started their careers before 1950. However, the same is not so for fWAR and ESPN. The overall finding of this sensitivity analysis is that conservatively weighting populations with respect to fan interest in baseball yields the conclusion as the analysis in Section 4: it is very unlikely that the pre-1950s time period could have produced so many historically great baseball players. 


\section{Additional comparison methods}

\subsection{Versus your peers methods}

There are several methods which are used to compare players across eras that do so by computing a baseline achievement threshold within one season and then comparing players to that baseline. These methods then rank players by how far they stood above their peers, the greatest players were better than their peers by the largest amount. We have shown that this approach can exhibit major biases in player comparisons as evidenced by career bWAR and fWAR. Adjusted OPS+ is a worse offender than bWAR or fWAR. Adjusted ERA+ is right in line with ESPN rankings.

\subsection{PPS detrending}

We describe and critique the methodology of Petersen et al. (2011) (PPS). As described in PPS, they detrend player statistics by normalizing achievements to seasonal averages, which they claim accounts for changes in relative player ability resulting from both exogenous and endogenous factors, such as talent dilution from expansion, equipment and training improvements, as well as performance enhancing drug usage. PPS misunderstands the effect of talent dilution from expansion and ignores reality. The talent pool was more diluted in the earlier eras of baseball than now because of a small relative eligible population size and the exclusion of entire populations of people on racial grounds. See Table 6 for the specifics. PPS's position with respect to equipment and training improvements is likewise not without fault because the same improvements are equally available to every competitor. Finally, PPS does not account for increases in salary compensation enjoyed by MLB players in modern eras, and their methodology fails to address segregation prior to 1947.

\begin{tabular}{ccccc}
\hline year & eligible pop. & number of teams & roster size & eligible pop. per roster spot \\
\hline 1890 & 5.01 & 8 & 15 & 41.7 \\
1910 & 8.56 & 16 & 25 & 21.4 \\
1930 & 9.92 & 16 & 25 & 24.8 \\
1950 & 11.59 & 16 & 25 & 29 \\
1970 & 24.49 & 24 & 25 & 40.8 \\
1990 & 37.46 & 26 & 25 & 57.6 \\
2010 & 72.27 & 30 & 25 & 96.4 \\
\hline
\end{tabular}

Table 6: Relative talent dilution when considering the MLB eligible population sizes at select time periods. Eligible population totals are in millions in column 2 and are in thousands in column 5.

The mathematics of PPS detrending is also questionable in the context of comparing baseball players 
across eras. PPS notes that the evolutionary nature of competition results in a non-stationary rate of success. They then detrend player statistics by normalizing achievements to seasonal averages. The normalization goes as follows: Suppose a player hits 40 homeruns in a given season and that the league average prowess for homerun hitting in that season is 10 homeruns. If the historical average prowess for homerun hitting is 5 homeruns then our player's detrended homerun count in that particular season is $40 \times(5 / 10)=20$. In general, the detrending formula is $Y \times$ (historic prowess/league prowess) where $Y$ is individual prowess for a particular player in a given season. We see PPS detrending as an inflationary metric of relative prowesses and not a detrending metric. Fundamentally different approaches for detrending are advocated in authoritative textbooks such as Introduction to Time Series and Forecasting, by Peter J. Brockwell and Richard A. Davis. Table 2 in PPS displays the top 25 career detrended homerun totals. It is clear that having higher prowess relative to your peers, hitting more runs in this case, is not indicative of a player's prowess with respect to peers from fundamentally different eras.

\subsection{Era bridging}

Berry et al. (1999) claim that their era bridging technique accounts for talent discrepancies across eras. However, they do not explicitly parameterize this in their hierarchical models. They state that "globalization has been less pronounced in the MLB (relative to other sports)... Baseball has remained fairly stable within the United States, where it has been an important part of the culture for more than a century" (Berry et al., 1999). This rationale ignores segregation, increases in the MLB eligible population relative to available roster spots, and increases in the average overall talent of that population. Therefore, there methodology does not fully address the characteristics of a changing talent pool.

In Berry et al. (1999, panel (c) of Figure 7) we see that their model predicts that a .300 hitter in 1996 will have a lower than .300 average for several seasons from 1900-1920. This conflicts with the well-established notion that the talent of baseball players has improved over time. In Berry et al. (1999, Table 9) we see that 6 of the 10 best hitters for average started their career before 1950 and 10 of the 25 best hitters for batting average started their careers before 1950. Their paper was published in 1999 so we recompute the chances of these events where the MLB eligible population ends at 1999. We calculate the chance that one would expect to observe 6 or more individuals in a top 10 list who started their careers before 1950 as 1 in 30 . We calculate the chance that one would expect to observe 10 or more individuals in a top 25 list who started their careers before 1950 as 1 in 7.7. These chances are not as extreme as those in Table 3, but they still 
correspond to events that are unlikely.

\section{Conclusions}

The MLB players from the early eras of baseball receive significant attention and praise as a result of their statistical achievements and their mythical lore. We find that these players are collectively overrepresented in rankings of the greatest players in the history of the MLB, and that popular performance metrics such as WAR fail to properly compare players across eras. Superior statistical accomplishments achieved by players that started their careers before 1950 are a reflection of our inability to properly compare talent across eras. It is highly unlikely that athletes from such a scarcely populated era of available baseball talent could represent top 10 and top 25 lists so abundantly.

We close with a general discussion on greatness. The conclusions of this article have broader implications than just rankings of baseball players. Who are the greatest all-time athletes in other sports, artists, musicians, actors and actresses, scientists, or leaders? Do our perceptions change when we focus beyond nostalgia?

\section{Acknowledgements}

This work would not be what it is today without the many conversations that the author had with family, friends, and peers. I am very grateful to Jim Albert, Peter M. Aronow, James Burrell, Xiaoxuan Cai, R. Dennis Cook, Forrest W. Crawford, Steven A. Culpepper, Andrew Depuy, Evan Eck, Fred Eck, Kim Eck, Marcus A. Eck, Michael Eck, Phil Eck, Shirley Eck, Wes Eck, Margret Erlendsdottir, Soheil Eshghi, Charles J. Geyer, Ed Kaplan, Zehong (Richard) Li, Adam Maidman, Aaron Molstad, Olga Morozova, Oliver Om, Kerry Purcell, Ken Ressel, Erick Ruuttila, Jesse Ruuttila, Anne Schuh, Bill Schuh, Yushuf Sharker, Ben Sherwood, Stephanna Szotkowski, Dootika Vats, Brandon Whited, the editorial board at Chance, and two anonymous referees at Chance for helpful comments and discussions (some of which went very long). This work was partially supported by NIH grants NICHD DP2 HD091799-01. 


\section{References}

Berry, S. M., Reese, C. S., and Larkey, P. D. (1999), "Bridging Different Eras in Sports," Journal of the American Statistical Association, 94, 447, 661-676.

Petersen, A. M., Penner, O., Stanley, H. E. (2011), "Methods for detrending success metrics to account for inflationary and deflationary factors," The European Physical Journal B, 79, 67-78.

Schmidt, M. B. and Berri, D. J. (2005), "Concentration of Playing Talent: Evolution in Major League Baseball," Journal of Sports Economics, 6, 412-419. 